\title{
LA INFORMÁTICA \\ Y LAS TELECOMUNICACIONES, SU UTILIDAD PARA LAS PYMES
}

Dra. BEATRIZ HERRERA GARCÍA

\section{INTRODUCCION}

Hoy en día no parece necesario convencer a nadie de las ventajas que para una empresa de cualquier tamaño supone una informatización bien dimensionada y acorde a las necesidades de la misma. Hasta la aparición del ordenador personal en el año 1982, la informatización de las empresas estaba muy condicionada a su tamaño, ya que el coste de un supercomputador o incluso un miniordenador los hacían prohibitivos. Con la aparición del ya bien conocido Personal Computer, la informática se ha extendido a todos los entornos de nuestra sociedad, incluido el residencial, donde cada día es mayor el número de hogares que posee un ordenador.

La fuerte evolución que se ha producido en las prestaciones de los ordenadores.junto con el abaratamiento de los mismos han provocado la aparición de programas con mayores posibilidades, de muy fácil manejo, y adecuados para casi cualquier tipo de aplicación que podamos necesitar. Hoy día es difícil encontrar empresas en las que en un alto porcentaje de su desarrollo no se haya adaptado, configurado o utilizado un producto ya disponible en el mercado, e.g., bases de datos, hojas de cálculo, control de procesos, etc.

En la actualidad existe un fenómeno similar a la evolución descrita de la informática y con un impacto muy importante sobre ésta. Nos referimos a las telecomunicaciones. No hace mucho tiempo, la interconexión de ordenadores y terminales dentro de una empresa mediante redes de área local o mediante diversas soluciones de distintos centros de éstas (centros de procesos de datos, sucursales, etc.) estaba restringida a entidades de un tamaño importante. Este hecho también está evolucionando de forma rápida y la utilización de las telecomunicaciones en forma integrada con programas informáticos está dejando de ser un aspecto restringido a grandes empresas. Hoy se puede observar que existen soluciones de muy bajo coste y unas altas prestaciones. Desde un simple módem hasta las altas prestaciones y calidad que ofrece la red digital de servicios integrados (el RDSI es una red de comunicaciones digitalizada y totalmente desplegada, que proporciona unas prestaciones superiores a las que presenta la red de telefonía básica), son soluciones asequibles tanto para profesionales independientes como para empresas de cualquier tamaño.

Estos acontecimientos han provocado una fuerte evolución en los programas informáticos. La integración de las telecomunicaciones en la informática corporativa hace unos años era escasa, existiendo aplicaciones informáticas dirigidas a la comunicación y otras únicamente orientadas al proceso de datos disponibles localmente. El término telemática era utilizado en ese momento para las técnicas de transferencia de datos que serían utilizados con posterioridad por aplicaciones informáticas. 
En la actualidad esto no es así, las telecomunicaciones son algo que, aunque imprescindibles ya que permiten procesar datos ubicados en ordenadores o terminales remotos, resultan trasparentes a las aplicaciones informáticas.

Con una apropiada integración informática-telecomunicaciones en una corporación se persigue que el usuario pueda usar diversas aplicaciones informáticas de forma uniforme en toda la organización y con abstracción total del lugar donde se encuentren o se generen los datos. Hoy día no se puede concebir y diseñar el soporte informático de una empresa de cualquier tamaño sin tener en cuenta las telecomunicaciones que vaya a emplear.

Algunos ejemplos muy extendidos y que siguen este esquema son las bases de datos distribuidas (bases de datos donde los datos se encuentran en cualquier punto de la red) o las aplicaciones cliente-servidor donde el procesamiento de los datos de la aplicación se puede llevar a cabo en cualquiera de los ordenadores conectados a la red con total trasparencia para el usuario, actuando como un verdadero sistema operativo distribuido.

Aunque en este artículo se pretende dar una visión de las ventajas que una apropiada informatización supone para una empresa y en especial para las PYMES, se hará especial hincapié en las nuevas oportunidades que la integración telecomunicaciones-informática ofrece. Un claro ejemplo es el fenómeno que ha supuesto en todos los sectores tanto empresariales como domésticos la aparición de Internet. Aunque existe una gran cantidad de documentación divulgativa y técnica al respecto o la que el lector se puede dirigir, se analizará aquí el alto valor añadido que para la pequeña y microempresa pueden ofrecer Internet. Este hecho se ve potenciado además por la rápida reducción de costes que se está produciendo en las tecnologías asociadas a este tipo de servicio.

La combinación de informática y telecomunicaciones abre, además, un nuevo espacio virtual para el comercio donde las empresas, sean del tamaño que sean, pueden ofrecer sus servicios y competir en igualdad de condiciones. Esto es posible gracias a los mínimos recursos que se precisan para entrar en este mercado y a la transferencia de costes que se consigue realizar a los clientes y proveedores.

Las consideraciones que deben tenerse en cuenta para una correcta integración de programas de diverso tipo con las telecomunicaciones corporativas independientemente de la tecnología de red utilizada, o mejor dicho algunas de las múltiples ventajas que la correcta informatización de una empresa puede ofrecer a ésta y que se pueden ver muy incrementadas con una apropiada integración con las telecomunicaciones de la corporación y todo ello a un coste muy reducido, lo que lo hace extensivo a las PYMES, son:

- Mejora de prodecimientos internos de trabajo de la empresa.- Este es probablemente el ejemplo que todo el mundo tiene más claro por ser quizás el origen de la informática en la empresa. Con la integración de las telecomunicaciones entre distintos centros de la empresa esta ventaja es extensible a toda la corporación. Este punto tiene además una ventaja adicional: permite reducir sustancialmente los recursos de la empresa dedicados a tareas rutinarias y permite concentrar dichos recursos en la actividad propia de la empresa, favoreciendo la creatividad y la iniciativa de sus trabajadores.

- Mejora en la relación con los clientes y proveedores.- El disponer de 
toda información de los clientes de una empresa "on line", o lo que es lo mismo, disponible en el momento en que exista cualquier relación con él, permite ofrecer una atención personalizada, lo que conducirá a una mejora sustancial de la imagen de la empresa. Por supuesto, una utilización conjunta de aplicaciones informáticas y comunicaciones permitirán ofrecer esta información "on line" a toda la corporación. Disponer de un sistema informatizado para la relación con los proveedores es tan importante como tener una relación ágil con los clientes. Con la ayuda de las telecomunicaciones podremos conseguir automatizar toda la gestión de pedidos, facturación, pagos, control de plazos de entrega, etc. lo que significará sin duda en un alto beneficio para el negocio. El hecho de poder llevar a cabo las relaciones de una empresa con sus clientes y proveedores de manera informatizada permite además una reducción importante de costes.

\section{- Nuevos canales de distribución.-}

Las redes de telecomunicaciones pueden ofrecer a las empresas un nuevo canal de distribución de productos. Pensemos en la gran cantidad de empresas que en la actualidad, anuncian y ofrecen sus productos a través de Internet. Términos como Telemárketing y Telemercado serán cada día más comunes para todos.

\section{- Mayor control del negocio me-} diante los sistemas de información.- La capacidad de obtener información de forma contínua de un proceso productivo que ofrecen los programas informáticos permite a la dirección de la empresa, disponer de una visión actualizada en todo momento de la situación del negocio. Pensemos en los esfuerzos requeridos en el pasado para realizar el balance de la empresa, en contraposición con las empresas modernas con un sistema de información totalmente informatizado donde se puede conocer la situación económica de ésta en cada momento.

- Clave en procesos de reingeniería y de calidad.- Es cada vez mayor el número de empresas sometidas a procesos internos de Reingeniería o de Gestión de la Calidad Total. Estos procesos van dirigidos a realizar un análisis permanente de los distintos procesos productivos de una empresa con objeto de conseguir una mejora contínua en los mismos. Estos procesos redundan en beneficios de distinta naturaleza para la empresa: aumento de la calidad, reducción de costes, reducción del tiempo de desarrollo de los productos, etc.

- La empresa sin papel.- Este es un objetivo que prácticamente todas las empresas persiguen hoy en día. Este aspecto sólo puede alcanzarse mediante la utilización de programas informáticos junto con unas telecomunicaciones adecuadas en todas las actividades de la empresa: compras, archivo documental, transferencia de información entre departamentos, relación con sus clientes y proveedores, etc.

En resumen, como se puede observar, son muchos los aspectos favorecidos en la empresa mediante una informatización apoyada y correctamente integrada en unas telecomunicaciones bien sean locales o de un ámbito amplio. Aunque se podrían seguir enumerando ventajas, podríamos resumir éstas en una ventaja competitiva para la empresa con todo lo que ello lleva consigo: aumento de la productividad, reducción de costes, aumento de la calidad, etc. 


\section{EL MODELO CLIENTE-SERVIDOR. 'APLICACIONES}

Otro aspecto clave a tener en cuenta al momento de decidir las aplicaciones informáticas de una determinada empresa, se relaciona con el grado de optimización del protocolo de intercambio de información.

Cuando dos aplicaciones se comunican entre sí, e.g., a través de internet, en las que un usuario accede a una base de datos remota o quiere trasferir un fichero a su computador desde un computador remoto, entran en:juego dos procesos diferentes. En el computador del usuario se ejecuta una aplicación denominada programa cliente que contacta con el computador remoto en el cual se ejecuta un programa denominado programa servidor. El programa cliente tiene dos funciones principales, por una parte, atender las peticiones del usuario y ofrecerle el resultado de sus consultas y, por otra, intercambiar información con el programa servidor que se ejecuta en el computador remoto. De esta forma, el programa servidor sólo necesita intercambiar información con los programas clientes que le estén consultando, liberándole así de todas las tareas relacionadas con la atención directa a los usuarios.

Se puede resumir que el modelo clienteservidor permite, gracias a la utilización de terminales de usuarios inteligente o PC (computadores personales) racionalizar la información que se transfiere entre los ordenadores locales y remotos; así como la carga de proceso de cada uno de ellos.

\section{- El concepto de aplicación distribuida.-}

Como extensión a los mecanismos que permiten intercambiar información entre distintas aplicaciones dentro de un computador, las aplicaciones informáticas han evolucionado de forma que consideran las redes de telecomunicaciones como nuevas vías de intercambio de información entre aplicaciones. Por ejemplo, en la actualidad la aplicación local considera al fichero remoto como un fichero local, de forma que al intentar llevar a cabo algún tratamiento del mismo el sistema lleva a cabo una transferencia automática del mismo totalmente transparente al usuario. Este es el verdadero concepto de aplicación distribuida.

Estos programas de computador son aplicaciones que una empresa usa en cualquier ubicación de ésta, sin que en muchas ocasiones sus usuarios sepan exactamente dónde se están ejecutando dichos programas. Por ejemplo, en una agencia bancaria cualquiera, cuando un empleado hace una consulta de una cuenta de ahorros de un cliente, sólo tiene que introducir el número de la cuenta y automáticamente recibirá la información deseada. En ningún momento el empleado es conciente de las múltiples transferencias que se han dado entre las aplicaciones cliente-servidor, entre el computador que está operando y el situado en el centro de cálculo del banco.

\section{- El concepto de empresa distribuida y} empresa virtual.- Extendiendo el ejemplo anterior a todos los procesos internos de una empresa, tal como ocurre hoy en día, se podría no tener que asociar distintas actividades interrelacionadas de una empresa con una proximidad geográfica de las unidades que las llevan a cabo. Gracias a la combinación de informática y telecomunicaciones se pueden ubicar las distintas unidades de una empresa en aquellos lugares que por uno u otro motivo (proximidad a los clientes o a las materias primas, etc.) se crean más convenientes. Esto es posible gracias a un sistema teleinformático que interconecta un departamento de la empresa con su sede central. 
Por tanto, ya no se puede identificar un lugar concreto con la actividad de la empresa, sino que la empresa se puede considerar distribuida, optimizando así sus recursos, gracias al uso de la informática y las telecomunicaciones.

Existe aún un grado más en la distribución de actividades de una empresa; cada vez son más las empresas con mayor grado de especialización y, por tanto, los costes que le supondrían a la propia empresa en el desarrollo de dichas actividades son menores. La relación entre la empresa contratista y contratada, utiliza en forma creciente el uso de sistemas teleinformáticos para dar soporte a la relación entre ambas.

Por otra parte, en un plano cada vez más actual y potenciado por las tecnologías informáticas, se encuentra una nueva forma de relación empresarial basado en la colaboración a un mismo nivel. El objetivo de esta estrategia está en la complementación mutua de actividades, lo que proporciona un valor agregado a los productos que se ofrecen, gracias a la sinergia de dicha acción conjunta. En definitiva, estas fórmulas permiten que con un tamaño de empresa relativamente pequeña y con un sistema teleinformático adecuado se pueda llevar a cabo una importante actividad, basada en el control de importantes recursos humanos y materiales, gracias a una relación ágil y rápida con un gran número de empresas ubicadas en cualquier punto del país o incluso del exterior.

El escenario actual mueve a estructuras cada vez más globalizadas, y por eso se puede afirmar que en esta nueva sociedad empresarial, desconcentrada e interconectada, van perdiendo significado los términos de grande, pequeña y mediana empresa, porque el triple ingrediente de creatividad, información y tecnologías de la información hace que cualquier empresa pueda abordar, trabajando en red, retos de cualquier envergadura.

Este es el concepto que se ha dado en llamar "empresa virtual". Imaginemos que este concepto no sólo es aplicable en la actualidad a la relación entre empresas para mantener relaciones de contratistasuministrador. Incluso en la relación directa con los clientes, actualmente, gracias a internet, un escaparate virtual donde cualquier empresa puede contactar con sus clientes directos a través de los medios de teleinformáticos. Se aprecia que gracias a las mayores opciones y prestaciones que ofrecen, la unión de la informática y las telecomunicaciones trata de sustituir en gran medida los mecanismos de relación directos actuales entre empresas y entre éstas y sus clientes, lo que redunda en una mayor reducción de costes y por tanto de competitividad.

\section{- El trabajo cooperativo:}

Las Telerreuniones.- Cada día es mayor el número de programas software orientados a ser utilizados de forma distribuida con objeto de facilitar el trabajo en grupo. Entre estas herramientas debe destacar aquellas que van dirigidas a permitir concretizar sesiones de trabajo de forma interactiva entre dos o más personas simultáneamente conectadas a la red. Es lo que se ha dado en llamar "teletrabajo en grupo" o "trabajo cooperativo" soportado por PCs. Estos programas tratan de proveer a los trabajadores de todas las herramientas necesarias para que puedan realizar reuniones de trabajo a través de un computador desde puntos distantes.

Entre las principales herramientas que ofrecen estos programas cabe destacar los siguientes: 
-Videoconferencias.- Ayuda a tener un contacto más directo entre las personas que participan en una sesión de trabajo cooperativo.

-Transferencia de ficheros.- Permite entregar documentos, fotografías, archivos, etc., al resto de participantes a través de la red y de forma instantánea.

-Pizarra compartida.- Es una zona de la pantalla del computador de cada participante que se usa de forma compartida entre todos como una mesa común de trabajo. Todo documento, texto, dibujo, etc., que se realice sobre esta zona por cualquiera de los participantes es visto de igual manera por el resto e, incluso, editable de manera conjunta.

-Compartición de aplicaciones.- Esta herramienta permite que cualquier aplicación o programa software que ejecuta cualquier participante en la teleconferencia, puede ser visto por todos con objeto de poder realizar determinados trabajos de forma conjunta.

-Telepuntero.- En algunas ocasiones se desea indicar a los participantes de una sesión de trabajo en grupo una parte de un documento marcándolo con el mouse de nuestro computador. Esto es lo que permite el telepuntero. Cualquier persona puede ver la posición del mouse de cualquier otro participante, identificándolo del resto mediante distintas formas de colores.

Existen otras herramientas específicas de determinados productos software (mensajería entre los participantes, diálogos mediante textos, etc.,) dirigidas a facilitar la realización de sesiones de trabajo en grupo entre personas situadas en lugares distantes. Por tanto, se pueden establecer verdaderas reuniones "virtuales" entre personas en puntos distantes gracias al desarrollo de la informática y las telecomunicaciones.

\section{TERMINOLOGÍA ESPECIALIZADA}

Se define a continuación una serie de términos mayormente usados y que pertenecen en general al mundo de la informática:

Correo Electrónico (e-mail). Aplicación informática que permite el envío y recepción de mensajes entre usuarios de computadores. Los mensajes enviados son almacenados en el computador de recepción y leídos en su momento por el destinatario.

Escáner. Dispositivo informático que permite registrar imágenes impresas y almacenarlas en un computador.

Internet. Conjunto de redes interconectadas y desplegadas por todo el mundo que proporciona todo tipo de información y permite la comunicación entre usuarios a través de una serie de aplicaciones definidas, tales como el correo electrónico (e-mail), transferencia de ficheros o el WWW (world wide web).

Módem (modulador-demodulador). Dispositivo que permite la conexión de un equipo informático a la red telefónica básica para su comunicación con otros equipos informáticos.

Navegador. Programa informático que permite el acceso al WWW desde un computador.

Página web. Cualquier pantalla de world wide web a la que se accede a través del navegador.

Software. Conjunto de programas y aplicaciones informáticas en su sentido más amplio. 
TQM (Total Quality Management). Gestión de Calidad Total. Conjunto de estrategias específicas destinadas a la mejora de los procesos internos de la empresa.

Trasferencia de ficheros. Aplicación informática que permite al usuario acceder desde un computador a otro remoto para extraer o dejar ficheros del mismo.

World Wide Web. Expresión inglesa que se podría traducir como "telaraña mundial". Aplicación estándar de internet que permite la visualización a través de la pantalla del computador, de información multimedia de la más variada naturaleza, ubicada en diferentes computadores desplegados por todo el mundo. 